\title{
Exploring the Relationship between Piano Playing Skills and Piano Sound
}

\author{
Jing LV \\ Northern National University, Yinchuan, Ningxia 750021, China
}

Keywords: Piano, playing skill, timbre.

\begin{abstract}
Piano has a very important position in the musical instrument family because of its loudness, wide range of sound, sound and intensity. Since the piano has been produced, whether it is classical, romantic, impressionist style of music, or modern jazz or rock music, piano is not only a composer, performers master music art, gallop in the infinite broad art world music art learning, research, processing and creation of basic tools, but also music workers to learn music theory knowledge, to create and play a good helper, or to promote the theory with practice, keyboard skills with the sound feel balanced development of the "experimental field." Beautiful and melodious unique timbre is one of the main reasons people love the piano, in the process of playing the piano, through different playing skills can produce different sounds, and then play a different music, get a different performance, to convey the piano player and piano tracks in different emotions and vitality. This article from the touch mode and the use of two skills pedal, exploring the relationship between playing skills and voices has important positive significance for piano players to improve their playing levels.
\end{abstract}

\section{Introduction}

The piano is the most widely used instrument in the world. In the kingdom of musical art, the piano is known for its loudness, wide range, sound and intensity, so the piano occupies a very important position in the musical instrument family. The piano can make an infinite, expressive voice. Whether it is rich colors of harmony, or lines of complex withered, and whether it is the wind-like clouds, or euphemism melody, or the momentum of the magnificent symphony, or warm and beautiful talk, the piano can be vividly performance. In addition to being an unparalleled solo instrument on the musical stage, the piano often plays an important role in the ensemble, ensemble and accompaniment. Piano can also be with the huge orchestra together, performing piano and band combination of symphony, concerto. Open the history of the world music, you can find a lot of composers have written in different forms, genre of piano. In the world music library, the number of piano music is the most, and its artistic charm enduring. People who study music also need to learn piano. Not only that, the piano as a multi-sound can play the keyboard instruments, but also by some composers as a music creation tool. Composers often streamline some of the complex music of the band into piano spectrum for easy playing and communication. Into the twentieth century, when some of the composer music reform, the piano is often used as a test of these composers. Because the piano has these excellent quality and irreplaceable artistic expression function, it is often said that the piano is "the king of instruments".

\section{The piano sound of the United States}

The sound is the reflection of the objective object auditory vibration of the instrument and the human voice object. Different sound body due to the structure, material, shape, composition of the different, the sound of the sound is also different, so through the sound can effectively distinguish between different sounding bodies. The sound is the color and the characteristic of the sound, the different sounding body (musical instrument or vocals), even if the same pitch and the same volume of the case, according to the different tone can distinguish between the different body. For the piano, the string vibration is not a simple harmonic vibration but a harmonic vibration, the strings were hit (excited) hair, and the strings produce many antinodes and nodes of the complex harmonic, the whole 
string Vibration and contain the segment vibration, which are the so-called piano wave (overtone) characteristics. So, try to play the piano in the bass area, often clearly hear the pitch and a series of homophonic sound at the same time sound. The frequency of the tone is determined by the pitch, the tone is mainly determined by the amount of overtone and its intensity, so the pitch frequency determines the pitch, and the number of overtone or harmonica and its intensity determines the sound. Tightly in the piano string of more than 200 strings and sound board, the coupling between the frameworks is the piano sound characteristics of the "original" conditions. The charm of the piano sound is in the coupling of these multiple factors, through the skillful playing and diversity of skills, many kinds of notes are combined, superimposed.

Among the many instruments, the piano bass is rich and powerful, the midrange is naturally smooth and the treble is bright and gorgeous. It is more harmonious with the human voice and various instruments. It is clear in the performance of the music, the ups and downs are large, the multi- , Flow sense, set off and rendering a strong role, easily lead to exciting results. Therefore, the piano can not only as a solo (concerto) form of the main theme of the instrument, but also solfeggio and vocal music, instrumental accompaniment good partner. In the film, drama, dance, gymnastics, music prose, poetry and other art, there is no piano sound, in the family, it is also competent to sing the companion.

Piano sound effect is loud, can create a magnificent atmosphere, the piano has a very wide range, in the modern music performing arts, can use the music sound of more than 90, and the piano 88 keys almost including the entire music soundtrack, both to show the vast world of music, the range from the weakest to the strongest intensity of the subdivision and can show a structured music expression, the player through the effective control of the piano sound, fully express their own emotions and their understanding of the repertoire and the degree of integration for the audience to create a different musical atmosphere, to show different musical charm, and for the listener, exposure to the colorful music hall, the brain with the music changes have a lot of imagination, you can enjoy and appreciate the beauty of art, to enhance the audience's artistic attainments at the same time, so that the audience in the spirit and mind to be relaxed and happy, cultivate the audience's sentiments and character, to express their own personal feelings and Insights on life.

\section{The development of piano playing skills}

Piano playing skills are very rich, can freely play a variety of scales, chromatic scale, a variety of interval beating, a variety of tones, chords and a variety of complex music. Piano playing skills have been developed for a long time, only through the piano sound device tuning tones to control the piano sound, resulting in piano tone ups and downs of small, the level is not clear, in the piano playing there is a single form of the problem, playing the effect of less than expected diversification, playing skills are relatively primitive. At the end of the nineteenth century, with the development of piano pronunciation devices, pianists could draw tones through harmonies, and piano sounds were also diversified due to changes in playing skills, and the control of the player was no longer limited to the adjustment, so that the piano can play the content, can convey the charm of music, can show the music world earlier changes have taken place. Today's piano playing skills have evolved over a century, and the progress of tuning technology has made the piano more diversified and diversified, making the piano more expressive and appealing. Through skilled use of piano playing skills, the player can convey to the audience the rich emotional changes in his heart, or pleasure, or sadness, or excitement, or frustration, triggering the emotional resonance of the audience.

\section{Touch the way and the relationship between the piano sounds}

Players through the touch keys to produce strings vibration, string vibration is a complex vibration, the strings were hit (excited) after the hair, the strings produce many antinodes and waves of complex harmonics, resulting in different sounds. The piano's touching skill is an important playing skill in piano playing. When playing piano, the player can use the correct piano touch method to play the flexibility and diversity of the tone, touch the action, skills, strength are the key factors affecting the 
piano sound. But many piano players are not too much attention to the piano touch the way, even if it has a certain level of performance and skillful playing skills, the performance of the sound will be the lack of flexibility and substituting sense, the stiff and inflexible timbre cannot highlight the unique charm of the piano.

(1) Touch key position

Touch the finger position refers to the contact with the piano keys and finger touch area, from the fingertips of the fingers to the pulp to the finger surface, different fingers touch the keyboard keys touch the location of the different will produce a different tone. If the player plays the piano with the pulp or the finger, the piano's playing intensity will be weakened, the pitch of the piano will be soft, the tone will change little, and if the piano is played with his fingertips, the piano playing intensity will be strengthened, the piano sound bias bright and brilliant.

(2) Touch the height

Touch height refers to the height of the contact between the finger and the keyboard, and the height of the touch key will affect the timbre of the piano's final sound. If the player enhances the height of the finger and the key, the piano's voice is bright and glorious, and if the finger is close to the key at a lower height and the process is slow and smooth, the piano's tone tends to be gentle and soft.

(3) Touch speed

Touch key speed refers to the speed of the finger touching the piano key, and the speed of touching key will affect the timbre of the piano. If the player touches the piano key at a faster speed, the piano's tone tends to be strong and strong, and if the piano keys are touched at a slower speed, the piano's tone tends to be weak and low.

(4) Touch the intensity

Touch the intensity refers to the fingers touch the moment the power of the piano keys, fingers touch the intensity of the fingers as the medium, the player's arm, arm and fingers on the power transmitted to the keys on the keys to repeat harmonic vibration, The size of the power of different piano sound produced by the sound there are differences. If the player touches the piano key in a horizontal manner, the piano's tone tends to be gentle, and if the piano keys are touched in a vertical way, the piano's voice tends to be sonorous.

Therefore, the player should perform the piano performance, should be based on the emotions to express, to convey the appeal, precise control of the finger touch position, touch the height, touch the speed and touch the intensity to produce the player expected Sound.

\section{The use of pedal and piano sound relationship}

The famous piano master Anton Rubinstein said that the pedal is the soul of the piano, in the piano playing process, the pedal of the use of the piano sound produced by the important and subtle, with the pedal of the rational use of techniques can strengthen the music The rhythm of the music, enhance the music of the harmony effect, so that players arbitrarily change the sound wave waveform to enrich the musical expression, to meet the needs of the hearing. Pedals are one of the important components of the piano, cannot exist as a separate technology, pedal use different methods will produce different sound effects, so the piano player should have a familiar ability to use the pedal in order to accurately control the pedal, more diverse sound, improve the piano sound of the appeal and expression.

In the piano playing, the pedal of the use of more methods, the use of methods are more flexible, the player can according to their own understanding of the repertoire and personal feelings to express at any time to change the pedal of the use of techniques, precise control pedal trampling and tread frequency, so that the pedal and the piano keys issued by the sound of mutual cooperation, mutual integration, rendering and playing the content and the emotions to be matched to match the music atmosphere. Therefore, in the use of pedal to give the piano sound appeal and expression before the need to play the repertoire to have a full understanding, which requires players to deep into the creation of the works of the times, to understand the creator of life experience and creative background, the content of the story in the repertoire, the artistic theme to be expressed, and the creative emotions contained in the work, and then use the familiar pedal to express the inner emotions 
contained in the work, like the film, slowly speak to the audience about the people and things that the creators have experienced, the joy and the loss, the resentment against the situation and the good imagination of the future. For example, during the Baroque period, the aristocracy was in power and the position of the musicians was given by the court. The musicians were responsible for providing music services for the courts and churches. Therefore, during the Baroque period, the works of the court musicians were mostly to cater to the upper classes such as the court and the church demand, musicians through exquisite delicate and gorgeous dazzling artistic style, hype and respected the palace of the magnificent, the palace of the extravagant extravagance, high society elegant, to please the court and the church's power. Baroque period of the famous piano composers are Germany's Bach, France's Coplan, etc., this period of composer with emotion as art point of view and aesthetic point of view, hoping to make the audience through the music emotional resonance, with the music works of emotional fluctuations and the minds of thousands, so the player playing Baroque piano tracks, it should reduce the use of pedals, through the piano's own voice to lead the audience to understand the emotional ups and downs, and reduce the pedal to bring the shock and appeal.

\section{Conclusion}

The piano is the best "teaching aids" to learn music. It is the best partner for musicians. People can talk about music on the keyboard of the piano, master the harmony, learn to play and even learn to play. Piano sound effect is loud, you can create a magnificent atmosphere, the range from the weakest to the strongest intensity of the subdivision, both can show a structured musical expression, but also can show the vast world of music. The performer of the piano sound comprehensive control through different playing skills, the full expression of the repertoire of understanding and integration, to create out of the ordinary music atmosphere for the audience, to show the charm of different music. In this paper, the relationship between the piano playing skills and the piano sound is analyzed, the player through the piano touch the precise control of the piano sound to ensure the beautiful sounds, through the rational use of the "pedal" skills, can greatly enrich the performance of music, which is composed of piano beautiful sound of the mystery lies. Players through the piano playing skills to fully control the piano sound, to meet the audience's auditory needs and artistic needs, so that the audience in the spirit and mind to be relaxed and happy, cultivate the audience's sentiments and character, to express their own personal feelings and life insights.

\section{Acknowledgements}

Lv Jing, female (1984.10----), Inner Mongolia, Bayannaoer, lecturer, Ph. D. in reading, research: piano performance and teaching. Correspondence address: Ningxia Yinchuan Northern Nationalities University Music and Dance Academy. Mobile: 13269228216

\section{References}

[1] Jin Yang. Exploring the Relationship between Piano Playing Skills and Piano Sound [J]. Academic periodical Piano (Journal of Shenyang Conservatory of Music), 2016, (04): 206-208.

[2] Yiyu Zhu. Analysis of the relationship between piano playing skills and piano timbre [J]. Art education, 2015, (11): 189.

[3] Ke Peng. The relationship between piano playing skills and piano timbre [J]. Music time and space, 2015, (09): 93.

[4] Jing Zhu. Analysis of the relationship between piano performance and piano timbre [J]. Journal of Career Academy, Jiamusi, 2014, (12): 157.

[5] Shufang Zhang. Analysis of the relationship between piano playing skill and piano timbre [J]. Journal of Wuzhou University, 2014, (04): 66-69. 